\title{
Predominance of an altered sense of smell or taste among long-lasting symptoms in patients with mildly symptomatic COVID-19*
}

\author{
Paolo Boscolo-Rizzo', Jerry Polesel², Giacomo Spinato', Cristoforo Fabbris'， Rhinology 58: 5, 524-526, 2020 \\ Leonardo Calvanese', Anna Menegaldo', Daniele Borsetto³, Claire Hopkins ${ }^{3}$ \\ https://doi.org/10.4193/Rhin20.263 \\ 'Section of Otorhinolaryngology, University of Padova, Treviso, Italy \\ *Received for publication: \\ ${ }^{2}$ Unit of Cancer Epidemiology, Centro di Riferimento Oncologico di Aviano (CRO) IRCCS, Aviano, Italy \\ May 29, 2020 \\ ${ }^{3}$ Guy's and St Thomas' Hospitals, London, United Kingdom \\ Accepted: June 26, 2020
}

\section{To the Editor:}

There is mounting evidence that a new onset of altered sense of smell or taste is related to severe acute respiratory syndrome coronavirus 2 (SARS-CoV-2) infection. In order to allow patients to recognize symptoms indicative of SARS-CoV-2 infection and self-isolate at the earliest opportunity, self-reported loss of smell and taste have greater value in controlling disease transmission than psychophysical testing, which is not widely available outside of highly specialized clinics.

We recently reported in the Journal of the American Medical Association the prevalence of COVID-19 related symptoms among a case series of mildly symptomatic home-isolated subjects with confirmed SARS-CoV-2 infection, with special emphasis to self-reported new onset of altered sense of smell or taste ${ }^{(1)}$. The same cohort of patients was closely monitored to assess the evolution of smell or taste loss as well as other COVID-19 related symptoms. Patient perception of sense of smell and taste are strongly associated and although both true olfactory and gustatory dysfunction have been demonstrated in COVID-19, when patients self-report, what they describe as loss of taste is often loss of flavor due to impaired retronasal olfaction (flavor) rather than true impaired gustatory function. We therefore evaluated these symptoms using SNOT-22 (Sino-nasal outcome test 22) item that combines assessment of both sense of smell and taste, as this reflects what is perceived and reported by patients themselves. Although self-rating of loss of smell appears to be less sensitive that psychophysical testing, patients do appear to be more reliable in reporting anosmia that they are in reporting normosmia ${ }^{(2)}$. Furthermore, the SNOT-22 "smell or taste" has indeed be shown to correlate with psychophysical testing of olfactory function using Sniffin' Sticks ${ }^{(3)}$.

The study was conducted with the approval of the institutio- nal ethical review board and informed consent was obtained verbally. After collecting clinical data through a survey administered by telephone interview near the onset of the disease, the same patients were re-contacted after 4 and 8 weeks. During the subsequent interviews the same questions were re-administered. In details, symptoms were assessed through ad hoc questions and structured questionnaires, including the ARTIQ (Acute Respiratory Tract Infection Questionnaire) and the SNOT-22, item "Sense of smell or taste", as previously reported (1). Patients were asked to rate impairment of smell or taste using a 6-point Likert scale, scoring 0 for no problem, to 5 for worst possible. Symptom prevalence was expressed as percentage of total patients, and $95 \%$ confidence interval $(\mathrm{Cl})$ were calculated using Clopper-Pearson method and differences in prevalence were evaluated through Fisher's exact test. Statistical analyses were performed using R 3.6.

Of 202 mildly symptomatic SARS-CoV-2 RNA positive adults ( $\geq 18$ years) consecutively assessed at Treviso Regional Hospital and completing the survey at baseline, two died after study enrollment and two needed hospitalization; 183 subjects (91.5\%) answered to both the 4-week and 8-week follow-up interview. We describe the evolution of COVID-19 related symptoms and sense of smell or taste in the 183 subjects who responded to all 3 interviews.

After 8 weeks from the baseline the most prevalent symptoms complained by patients were: altered sense of smell or taste (18.6\%, 95\% Cl: 13.2\%-25.0\%), fatigue (13.1\%, 95\% Cl: 8.6\%$18.9 \%)$, breathing problems (10.4\%, $95 \% \mathrm{Cl}: 6.4 \%-15.7 \%)$, and muscle or joint pains (7.7\%, 95\% Cl: 4.2\%-12.5\%) (Table 1). Figure 1 shows the evolution and intensity of loss of smell or taste at baseline and during the follow-up. Among 34 subjects com- 
Table 1. Symptom's evolution from baseline to the 8-week follow-up in 183 patients positive for SARS-CoV-2.

\begin{tabular}{|c|c|c|c|c|c|c|}
\hline \multirow[t]{3}{*}{ Symptoms } & \multicolumn{6}{|c|}{ Symptom's prevalence } \\
\hline & \multicolumn{2}{|c|}{ Baseline } & \multicolumn{2}{|c|}{ 4-week follow-up } & \multicolumn{2}{|c|}{ 8-week follow-up } \\
\hline & $\mathbf{n}$ & $\%(95 \% \mathrm{Cl})$ & $\mathbf{n}$ & $\%(95 \% \mathrm{Cl})$ & $\mathbf{N}$ & $\%(95 \% \mathrm{Cl})$ \\
\hline Fever & 101 & $55.2(47.7-62.5)$ & 8 & $4.4(1.9-8.4)$ & 0 & $0.0(0.0-2.0)$ \\
\hline Dry cough or coughing up mucus & 115 & $62.8(55.4-69.9)$ & 53 & $29.0(22.5-36.1)$ & 5 & $2.7(0.9-6.3)$ \\
\hline Blocked nose & 69 & $37.7(30.7-45.2)$ & 28 & $15.3(10.4-21.3)$ & 2 & $1.1(0.1-3.9)$ \\
\hline Problems breathing & 76 & $41.5(34.3-49.0)$ & 44 & $24.0(18.0-30.9)$ & 19 & $10.4(6.4-15.7)$ \\
\hline Headache & 77 & $42.1(34.8-49.6)$ & 23 & $12.6(8.1-18.3)$ & 5 & $2.7(0.9-6.3)$ \\
\hline Sore throat & 57 & $31.1(24.5-38.4)$ & 13 & $7.1(3.8-11.8)$ & 5 & $2.7(0.9-6.3)$ \\
\hline Muscle or joint pains & 83 & $45.4(38.0-52.9)$ & 30 & $16.4(11.3-22.6)$ & 14 & $7.7(4.2-12.5)$ \\
\hline Chest pain & 28 & $15.3(10.4-21.3)$ & 11 & $6.0(3.0-10.5)$ & 2 & $1.1(0.1-3.9)$ \\
\hline Sinonasal pain & 31 & $16.9(11.8-23.2)$ & 5 & $2.7(0.9-6.3)$ & 2 & $1.1(0.1-3.9)$ \\
\hline Loss of appetite & 100 & $54.6(47.1-62.0)$ & 20 & $10.9(6.8-16.4)$ & 5 & $2.7(0.9-6.3)$ \\
\hline Felt tired & 126 & $68.9(61.6-75.5)$ & 29 & $15.8(10.9-22.0)$ & 24 & $13.1(8.6-18.9)$ \\
\hline Diarrhoea & 83 & $45.4(38.0-52.9)$ & 11 & $6.0(3.0-10.5)$ & 2 & $1.1(0.1-3.9)$ \\
\hline Nausea & 36 & $19.7(14.2-26.2)$ & 2 & $1.1(0.1-3.9)$ & 0 & $0.0(0.0-2.0)$ \\
\hline Vomit & 12 & $6.6(3.4-11.2)$ & 1 & $0.5(0.0-3.0)$ & 0 & $0.0(0.0-2.0)$ \\
\hline Abdominal pain & 23 & $12.6(8.1-18.3)$ & 7 & $3.8(1.6-7.7)$ & 0 & $0.0(0.0-2.0)$ \\
\hline Dizziness & 25 & $13.7(9.0-19.5)$ & 5 & $2.7(0.9-6.3)$ & 5 & $2.7(0.9-6.3)$ \\
\hline Altered sense of smell or taste & 110 & $60.1(52.6-67.3)$ & 67 & $36.6(29.6-44.0)$ & 34 & $18.6(13.2-25.0)$ \\
\hline
\end{tabular}

SARS-CoV-2: severe acute respiratory syndrome coronavirus 2.

plaining of smell or taste impairment at the 8 weeks follow-up, 33 were found to be SARS-CoV-2 negative at control swab test performed between 4-8 weeks after the initial swab. One patient that only complained of cough and breathing problems at baseline and developed grade 4 smell or taste impairment between the 4- and 8-week follow-up, was found still SARS-CoV-2 positive at control swab test performed between the 4 and 8 weeks after the initial swab. No symptoms at baseline were found to be predictive of persistent loss of smell and taste at 8 weeks. Moreover, no association were observed between smell or taste impairment at baseline and its persistence at 8 weeks.

Long-lasting fatigue was significantly associated with persistent altered sense of smell or taste $(p=0.007)$. Among patients complaining of loss of sense of smell or taste at baseline, 4 weeks, and 8 weeks, 37.3\% (41/110), 19.4\% (13/67), and 2.9\% (1/34), respectively, also complained of a blocked nose, with this symptom being significantly associated with altered chemosensory function only at baseline $(p=0.011)$. Among the 41 cases complaining of loss of smell or taste associated with blocked nose at baseline, only 17 have the resolution of smell or taste impairment concomitant with the resolution of blocked nose during the follow-up.
The following conclusions can be drawn from these observations. We have shown previously that in patients with SARSCoV-2 infection an altered sense of smell or taste is among the most common symptoms present at baseline ${ }^{(1)}$. Although most patients recovered from loss of smell and taste, this remains the most prevalent long-lasting symptom followed by fatigue and breathing problems. No symptoms at baseline are predictive of persistently altered chemosensory function. While other studies have recruited patients at different time points in their disease, and have estimated recovery rates ${ }^{(4-6)}$, this is the first study to report prospective self-rated loss of sense of smell at taste over 3 time points, at 0, 4 and 8 weeks. This confirms a high rate of recovery, with the prevalence of loss of smell and taste reducing from $60 \%$ to $19 \%$ of the cohort over 8 weeks of follow-up. Nonetheless, 1 in 5 perceive ongoing deficits in smell and taste and this may result in significant numbers of patients seeking medical care. Importantly, and for the first time found in the literature we demonstrate that patients may develop loss of smell and taste later in the course of their disease.

Unlike some other studies, we did find an association between nasal obstruction and olfactory dysfunction at baseline. We believe that this may relate to capturing patients very early in the course of COVID illness, where viral rhinitis may add a conductive component to the loss of sense of smell. We found no as- 


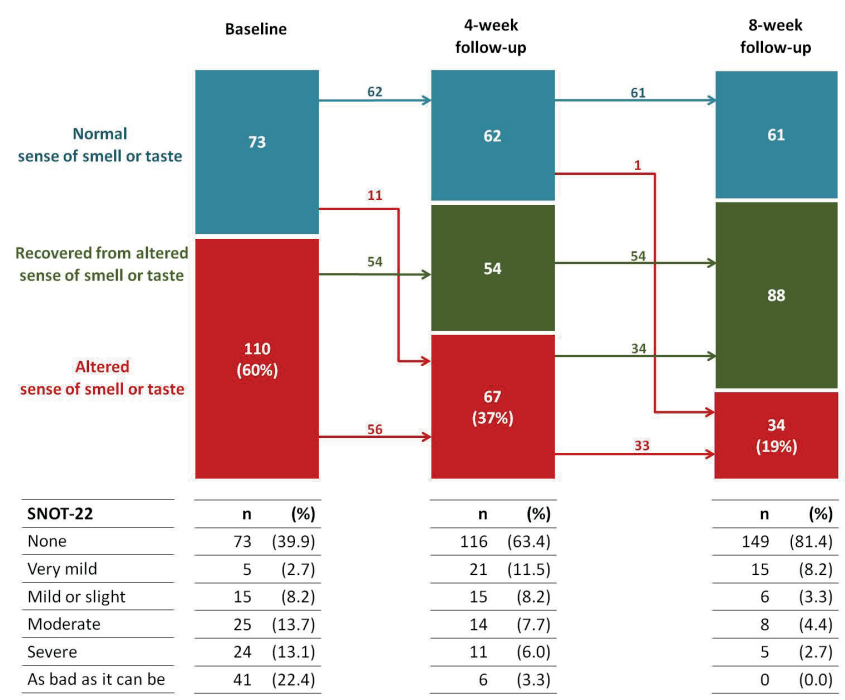

Figure 1. Prevalence and intensity of altered sens of smell or taste at baseline and during the follow-up.

sociation between resolution of nasal obstruction and olfactory function at 4 and 8 weeks consistent with the suggestion that in those with a loss persisting for 4 weeks or more, the aetiology is more likely to be due to sensorineural loss relating to injury to the olfactory epithelium or olfactory bulb.

While the new onset of smell or taste loss is associated with active SARS-CoV-2 infection, the persistent of smell or taste impairment is not associated with persistent detection of viral load, and patients can be reassured that they do not remain infectious.

\section{Conflict of interest}

None.

\section{Authorship contribution}

PBR and $\mathrm{CH}$ has led on the concept and written the initial draft. PBR had full access to all of the data in the study and take responsibility for the integrity of the data and the accuracy of the data analysis. GS, CF, LC, and AM collected patient's data. JP performed the statistical analysis. DB and JP helped writing the manuscript. PBR, CH, DB, and JP reviewed final draft.

\section{Acknowledgement}

The authors gratefully thank patients for their participation in this study.

\section{References}

1. Spinato, Giacomo, Fabbris C F Cristoforo, Polesel J P Jerry, et al. Alterations in Smell or Taste in Mildly Symptomatic Outpatients With SARS-CoV-2 Infection. Alter Smell Taste Mildly Symptomatic Outpatients SARSCoV-2 Infect. 2020;

2. Lötsch J, Hummel T. Clinical Usefulness of Self-Rated Olfactory Performance-A Data Science-Based Assessment of 6000 Patients. Chem Senses. 2019;44(6):357-64.

3. Ta NH, Hopkins C, Vennik J, Philpott C Optimising trial outcomes and patient retention for the MACRO trial for chronic rhinosinusitis. Rhinology. 2019;57(5):358-66.

4. Lechner M, Chandrasekharan D, Jumani K, et al. Anosmia as a presenting symptom of SARS-CoV-2 infection in healthcare workers - A systematic review of the literature, case series, and recommendations for clinica assessment and management. Rhinology. 2020; 58: 4, 394 - 399

5. Ottaviano G, Carecchio M, Scarpa B Marchese-Ragona R. Olfactory and rhinological evaluations in SARS-CoV-2 patients complaining of olfactory loss. Rhinology. 2020; 58: 4, 400 - 401

6. Hopkins C, Surda P, Kumar N. Presentation of new onset anosmia during the COVID-19 pandemic. Rhinology. 2020; 58: 3, 295 - 298.

Daniele Borsetto

Guy's Hospital London SE1 9RT

United Kingdom

Tel: +44 7853222040

E-mail: daniele.borsetto@gmail.com 